\title{
Evaluación del Efecto de un Gel con Arginina sobre el PH y Flujo Salival
}

\author{
Evaluation of the Effect of Arginine Gel on pH and Salivary Flow
}

\author{
Juan Gerardo Sánchez Medina1; Othoniel Hugo Aragón Martínez; \\ Juan Antonio Cepeda Bravo ${ }^{3}$ \& Saray Aranda Romo ${ }^{1}$
}

\begin{abstract}
SÁNCHEZ, M. J. G.; ARAGÓN, M. O. H.; CEPEDA, B. J. A. \& ARANDA, R. S. Evaluación del efecto de un gel con arginina sobre el PH y flujo salival. Int. J. Odontostomat., 15(4):989-996, 2021.

RESUMEN: Se evaluó el efecto de un gel con arginina sobre el pH y flujo salival después de un uso de catorce días en mujeres con desmineralización dental leve. Se estableció un piloto de ensayo clínico controladoen el cual fueron incluidas 20 mujeres de 18 -23 años, sistémicamente sanas y con al menos un órgano dental con desmineralización ICDAS 3 , dos grupos: Grupo A / sin arginina $(\mathrm{N}=10)$ y Grupo B/ con arginina ( $\mathrm{N}=10)$. Se realizó una evaluación clínica y toma de una muestra de saliva no estimulada para la determinación del pH, y la medición del flujo salival al inicio y 15 días posterior a la utilización del gel. Se realizó el análisis estadístico con el programa GraphPadPrism versión 8 . Una p<0,05 fue considerado como estadísticamente significativo. En ambos grupos se mantuvo el pH salival cercano a la neutralidad sin diferencias estadísticamente significativas y el flujo salival permaneció en valores normales tras la utilización del gel durante 14 días, aunque se observaron diferencias estadísticas significativas en la comparación inter-grupo. La utilización de un gel con arginina durante 14 días mantuvo el pH neutro y el flujo salival en niveles normales sin diferencias estadísticamente significativas con el grupo control.
\end{abstract}

PALABRAS CLAVE: caries, arginina, prebióticos, saliva, concentración de iones de hidrógeno, flujo salival.

\section{INTRODUCCIÓN}

La caries es un problema de salud pública a nivel mundial se caracteriza por una disbiosis microbiómica con elevación de bacterias cariogénicas, lo que lleva a la desmineralización de la superficie del esmalte (Lamont et al., 2018; Zhan, 2018).

Múltiples factores de riesgo contribuyen al desarrollo de la caries, al ser la cavidad oral un sistema abierto y dinámico, puede verse afectado por varios conductores de la enfermedad (concentración de oxígeno, el $\mathrm{pH}$, la dieta, el flujo salival, trastornos sistémicos, los hábitos de higiene oral, entre otros) lo que lleva a un cambio en el equilibrio entre comensales y patógenos, y el crecimiento excesivo de los agentes patógenos, favoreciendo el desarrollo de una disbiosis y enfermedades orales, como la caries (Zaura et al., 2014; Krishnan et al., 2017; Abe et al., 2018). El tratamiento dental restaurador no altera la carga de bacterias cariogénicas y no busca mantener la homeostasis (Zhan). Con estos conocimientos, muchos investigadores y multinacionales han desarrollo y aplicado la nueva ciencia emergente del microbioma oral para desarrollar alternativas preventivas y terapéuticas anticaries basadas en recuperar el equilibrio del microbioma oral y mantener la homeostasis; formulaciones "bioeficaces", prebióticos, probióticos, el uso de componentes nutricionales, homeopáticos, microminerales e ingredientes herbales son algunos ejemplos (Lin et al., 2018).

Los prebióticos son sustratos no viables que sirven como nutrientes para los microorganismos beneficio-

\footnotetext{
${ }^{1}$ Departamento de Microbiología y Patología Oral, Facultad de Estomatología, Universidad Autónoma de San Luis Potosí, S.L.P, México.

${ }^{2}$ Departamento de Farmacología, Facultad de Medicina, Universidad Autónoma de San Luis Potosí, S.L.P, México.

${ }^{3}$ Departamento de Periodoncia, Facultad de Estomatología, Universidad Autónoma de San Luis Potosí, S.L.P, México.
} 
sos que alberga el huésped, incluidas las cepas probióticas administradas y los microorganismos residentes (Monteagudo-Mera et al., 2019; Zaura \& Twetman, 2019). En la odontología, específicamente para el manejo de la caries y desmineralización, estos incluirían nutrientes para taxones microbianos que inhiben microorganismos acidógenos y acidúricos y / o mejoran la recuperación del pH salival al generar álcali a partir de estos nutrientes. Las dos fuentes principales de álcali en la cavidad oral son urea y arginina (Zaura \& Twetman).

La arginina es un aminoácido condicionalmente esencial en adultos sanos y, por lo tanto, no existe un requerimiento nutricional específico (Nascimento, 2018). Con el uso como prebiótico de la arginina, se ha demostrado que se equilibra el microbioma en disbiosis, ya que Streptococcus gordonii, Streptococcus sanguis, Streptococcus dentisani (bacterias orales que se asocian con un microbioma compatible con la salud) la metabolizan por la vía de la arginina deiminasa (ADS) produciendo citrulina, ornitina, $\mathrm{CO}_{2}$, adenosina trifosfato (ATP) y amoníaco. La producción de amoníaco a partir del metabolismo de la arginina produce un aumento del $\mathrm{pH}$ citoplasmático y en el ambiente oral, sirviendo como mecanismo utilizado por las bacterias orales para: 1) La protección contra la muerte celular, 2) Ventajas bioenergéticas, incluido el aumento de $\mathrm{pH}$ y la síntesis de ATP, 3) Mantener un $\mathrm{pH}$ salival relativamente neutral que favorece la persistencia de las bacterias ADS-positivas (ADS +) mientras es competitivo contra los patógenos de caries. Además, se promueve la remineralización del esmalte (Nascimento; Bijle et al., 2019; Monteagudo-Mera et al.).

Diferentes estudios han buscado establecer la relación pH salival - arginina: Un estudio in vivo, el cual investigó los efectos neutralizantes de un enjuague con bicarbonato de arginina sobre la disminución inducida por sacarosa en el $\mathrm{pH}$, encontró que el uso regular de un enjuague con arginina después del consumo de carbohidratos podría ayudar a prevenir la caries ya que después de su uso hay una neutralización inmediata en el pH (los valores se aproximan a 7) (Wang et al., 2012).

Otro estudio investigó el efecto de la suplementación dietética con arginina $(3 \mathrm{~g})$ sobre el $\mathrm{pH}$ salival, encontrando que la magnitud del cambio de $\mathrm{pH}$ fue significativamente mayor para el grupo de estudio que para el control, los autores recomiendan realizar más estudios para determinar los efectos de suplementos como la arginina sobre la composición bioquímica y pH de la saliva (Vuletic et al., 2013).
Con base en lo anteriormente descrito se propuso la evaluación de un gel con arginina al $8 \%$ sobre el $\mathrm{pH}$ y flujo salival a corto plazo, los datos generados son confiables y no ligados a una empresa multinacional.

\section{MATERIAL Y MÉTODO}

Población: Se realizó un piloto de un ensayo clínico controlado. El reclutamiento de los sujetos participantes, así como de la toma de muestras se realizó en el departamento de Diagnóstico de la Facultad de Estomatología de la UASLP. San Luis Potosí, México. Se incluyeron un total de 20 pacientes del sexo femenino, todas ellas estudiantes de odontología y con un rango de edad de 18 a 23 años en el periodo comprendido entre enero y marzo de 2020; mediante un muestreo por conveniencia.

Criterios de inclusión: Sexo Femenino, adultos jóvenes 18 a 23 años, que aceptaron participar en el estudio mediante la firma del consentimiento informado, presentes los 4 primeros molares, presencia de Caries ICDAS II - 3, sistémicamente sanas y con un cepillado mínimo de 2 veces al día.

Criterios de exclusión: Sujetos bajo un proceso infeccioso bucal o sistémico (con o sin tratamiento), sujetos fumadores, sujetos que hayan recibido terapia antibiótica en menos de 1 mes, sujetos que hayan usado alguna pasta dental y/o suplemento alimenticio con arginina en los últimos 6 meses.

Asignación de Grupos: Los grupos se formaron mediante un programa de computadora generador de grupos aleatorios. Los sujetos fueron divididos Grupo A Control / Gel sin arginina (10 sujetos), Grupo B Experimental/ Gel Arginina (10 sujetos). Para la elaboración del gel se tomó en cuenta una formulación utilizada por Ferrer et al. (2019) para evaluar la colonización oral y amortiguación del pH por el Streptococcus dentisani. Se prepararon dos presentaciones diferentes uno con goma guar y otro con goma guar más arginina; esto para poder establecer una diferencia entre los grupos control y experimental (Tabla I).

Tabla I. Composición experimental del gel.

\begin{tabular}{lc}
\hline Producto & $\begin{array}{c}\text { Composición del vial una vez mezclado } \\
\text { con } 10 \mathrm{ml} \text { de agua. }(\mathrm{g})\end{array}$ \\
\hline Goma Guar & 0,25 \\
Arginia & 0,8 \\
\hline
\end{tabular}


Plan de trabajo:El estudio fue aprobado previamente por el comité de ética de la Facultad de Estomatología bajo el folio CEI-FE-060-019. Se les informó a las pacientes el procedimiento a realizar y se les solicitó que en caso de aceptar firmaran el consentimiento informado. Posteriormente, se realizó el odontograma de cada paciente usando ICDAS II y el DIAGNO dent pen 2190 (KaVo Dental $®$, Bismarckring 39 Germany) un detector de caries con láser fluorescente como auxiliar diagnóstico (Proclinic, 2018), además de un cuestionario para confirmar que se cumpliera con los criterios de inclusión y exclusión, así como de una encuesta en línea con la herramienta Google Formularios que abordaba cuestiones relacionadas a signos y síntomas orales. Las pacientes que cumplieron con los criterios de inclusión se les tomaron impresiones con alginato de ambas arcadas para la confección de unas guardas de acetato del 0,25 blandas, se les solicitó acudir al día posterior, para la recolección de muestras iniciales. Se les dió como indicación evitar el cepillado durante 24 horas previas, y acudir a la cita en ayunas antes de las 10:00 am para que los alimentos no interfirieran. Además de que se les indicó llevar su cepillo dental.

Toma de muestra: Se llevó a cabo la toma de la muestra de saliva no estimulada según el protocolo derivado de la guía de la Organización Mundial de la Salud / "Normas y protocolos técnicos mínimos comunes" (International Agency for Research on Cancer, 2017) las pacientes recibieron agua potable (embotellada) y se

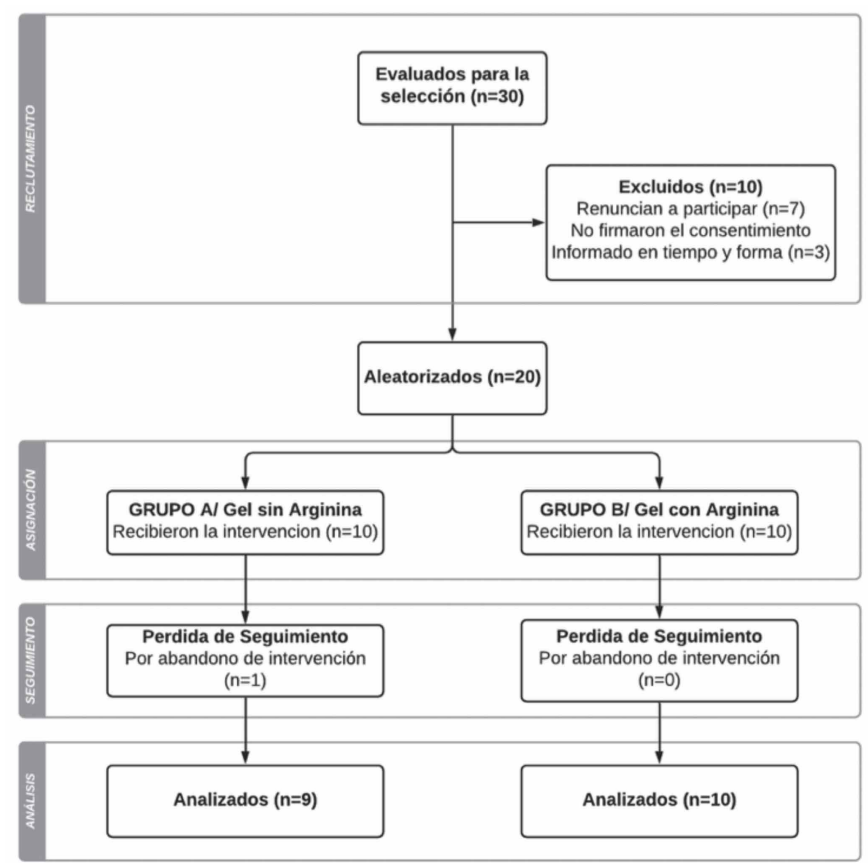

Figura 1. Evaluación del efecto de un Hidrogel con arginina sobre el $\mathrm{pH}$ y flujo salival. les pidió que se enjuagaran bien la boca, 5 minutos después de este enjuague, se les pidió que escupieran la saliva total. Se les pidió que se abstuvieran de hablar, que bajaran la cabeza y dejaran que la saliva corriera naturalmente hacia el frente de la boca, en el tubo de recolección aproximadamente una vez por minuto durante 5 minutos. Se recolectaba entre las 8:00 a.m. y las 10:00 a.m. Se registró la cantidad de saliva en $\mathrm{ml}$ según la graduación del tubo recolector. Por último, se instruyó a las pacientes sobre la técnica de cepillado correcta, se les indicó como usar el prebiótico en gel: "Se colocará el gel en las guardas de acetato, se colocará diariamente $1 \mathrm{ml}$ de la jeringa dispensadora, estas se utilizarán de manera nocturna. A la mañana siguiente retirar y realizar un enjuague con agua purificada, previo al cepillado." También se les dio una crema dental comercial, la misma para ambos grupos, el cepillado se realizó 3 veces al día, mañana, tarde y noche.

Procesamiento de Muestras: La medición de $\mathrm{pH}$ se realizó dentro de los primeros 30 minutos de su toma con un $\mathrm{pH}$ metro manual $\mathrm{pH}$ meter MODEL PH-02, Lohand Biological Co. Ltd.). El medidor de $\mathrm{pH}$ fue calibrado todos los días utilizando tampones recién preparados de $\mathrm{pH} 7$ y $\mathrm{pH} 4$. El valor arrojado se registró en una bitácora.

Segunda toma de muestra: La segunda toma de muestras se efectuó después de 2 semanas. Las pacientes fueron dotadas del gel a lo largo de seis semanas, al término de este período se volvió a aplicar la encuesta sobre signos y síntomas orales.

Análisis estadístico: Los datos demográficos y clínicos fueron analizados mediante estadística descriptiva e inferencial. Los datos para el $\mathrm{pH}$ y flujo salival fueron analizados con la prueba estadística correspondiente, mencionada en los resultados. Se utilizó el software GraphPadPrism versión 8.0.

\section{RESULTADOS}

\section{Características demográficas}

Fueron incluidas un total de $\mathrm{n}=20$ mujeres, las cuales aleatoriamente fueron asignadas a dos grupos para llevar a cabo la intervención. Grupo A (control/gel) Grupo B (experimental/gel + arginina). El diseño para llevar a cabo las diferentes fases del estudio se realizó de acuerdo a las guías CONSORT y se representan en la Figura 1. 
Ninguna de las pacientes incluidas reportó efectos adversos al uso del gel. En la Tabla II se muestran las características demográficas de la población de acuerdo con el grupo, en donde se observa que no hubo diferencias con respecto a la edad entre los grupos. Es importante mencionar que solo fueron incluidas mujeres debido a las diferencias en el microambiente oral con respecto al género. La mayoría de las pacientes $(80 \%)$ se encontraba estudiando octavo semestre de la carrera de médico estomatólogo.

\section{Características clínicas}

Fueron recolectadas las características clínicas como la presencia de Caries según el ICDAS II, las restauraciones presentes, frecuencia en la higiene oral (número de veces que se realiza en un día). Con respecto a la dieta solo se evaluó la ingesta de carbohidratos, y se consideró también la vía de nacimiento. Estas variables odontológicas, dietéticas y perinatales influyen en la adquisición y desarrollo del microbioma oral y tienen un impacto directo en la composición del microambiente oral (Tabla III).

Para evaluar caries se utilizaron los criterios clínicos basados en el ICDAS II, se identificó que todas las pacientes tenían al menos un órgano dental con ICDAS II- 3. Con la finalidad de obtener una medición más objetiva con respecto al grado de desmineralización del órgano dental se utilizó el Diagnodent y se observó que no hubo diferencias estadísticamente significativas entre los grupos con respecto a este valor en la medición inicial, por lo que ambos grupos fueron homogéneos en el grado de desmineralización dental. Con respecto al número de restauraciones presentes al momento de la evaluación se observó que no hubo diferencias entre los grupos. La higiene oral fue óptima ya que en ambos grupos el cepillado dental se realizaba 2 veces al día en promedio sin diferencias estadísticamente significativas. Finalmente se evaluó la ingesta de carbohidratos y la vía de nacimiento y se encontró que ambos grupos tenían una ingesta de carbohidratos regular sin diferencias significativas. El parto natural (vaginal) fue la vía de nacimiento más frecuente en ambos grupos.

Determinación del pH y flujo Salival previo y posterior a la utilización del gel: Se realizó la medición del $\mathrm{pH}$ a partir de las muestras de saliva obtenidas (inicial y una semana posterior a la utilización del gel). En la Tabla IV se muestra la comparación de los valores obtenidos del $\mathrm{pH}$ salival entre los grupos y en la Tabla $\mathrm{V}$ la comparación intragrupos. Se encontró que hubo una variación mínima en el pH en ambos grupos y se mantuvo con tendencia hacia la neutralidad.

Tabla IV. Comparación del pH salival intergrupo al inicio y final del tratamiento.

\begin{tabular}{llll}
\hline & Grupo A & Grupo B & P Value \\
\hline pH inicial $^{*}$ & $7,03 \pm 0,71$ & $6,98 \pm 0,60$ & $0,8843 \oplus$ \\
pH final $^{*}$ & $7,26 \pm 0,51$ & $6,97 \pm 0,29$ & $0,1732 \mathrm{~T}$ \\
\hline
\end{tabular}

*Media \pm SD según número de participantes. $\oplus$ Mann-Whitney test, $\mathrm{T}$ t-student

Tabla II. Características por Grupo de Estudio.

\begin{tabular}{lcccc} 
& Grupo A & Grupo B & Total $(=19$ & P Value \\
\hline Mujeres, $\mathrm{n}$ & 9 & 10 & 19 & \\
Edad, años $^{*}$ & $20,89 \pm 1,1$ & $21,7 \pm 1,1$ & $21,31 \pm 1,3$ & 0,4187 \\
\hline
\end{tabular}

*Media \pm SD según número de participantes.

Tabla III. Características por Grupo de Estudio.

\begin{tabular}{|c|c|c|c|c|c|}
\hline & \multicolumn{2}{|c|}{ Grupo A } & \multirow{2}{*}{$\begin{array}{l}\text { Grupo B } \\
\text { Edad, años* }\end{array}$} & \multirow{2}{*}{$\begin{array}{l}\text { Total }(=19 \\
20,89 \pm 1,1\end{array}$} & \multirow{2}{*}{$\begin{array}{l}P \text { Value } \\
0,1659\end{array}$} \\
\hline Caries* • & & $0,17 \pm 0,06$ & & & \\
\hline Restauraciones* & & $3,44 \pm 2,45$ & $2,6 \pm 2,93$ & $3 \pm 2,75$ & 0,5313 \\
\hline Frecuencia Higiene Oral ${ }^{*}$ & & $2,77 \pm 0,41$ & $2,5 \pm 0,8$ & $2.63 \pm 0,66$ & 0,3921 \\
\hline \multirow{3}{*}{ Ingesta de Carbohidratos* } & Baja & $1 \mid 11,11 \%$ & $1 \mid 10 \%$ & 2 | $10,52 \%$ & \multirow{3}{*}{0,6902} \\
\hline & Regular & $7 \mid 77,77 \%$ & $7 \mid 70 \%$ & $14 \mid 73,68 \%$ & \\
\hline & Alta & $1 \mid 11,11 \%$ & $2 \mid 20 \%$ & $3 \mid 15,78 \%$ & \\
\hline \multirow{2}{*}{ Vía de Nacimiento* } & Vaginal & $6 \mid 66,66 \%$ & $6 \mid 60 \%$ & 12 | $63,15 \%$ & \multirow{2}{*}{0,7789} \\
\hline & Cesárea & $3 \mid 33,33 \%$ & $4 \mid 40 \%$ & $7 \mid 36,84 \%$ & \\
\hline
\end{tabular}

*Media \pm SD según número de participantes $•$ Media según escala de ICDAS y superficies analizadas. 
Tabla V. Comparación del pH salival intragrupo al inicio y final del tratamiento.

\begin{tabular}{|c|c|c|c|}
\hline & $\mathrm{pH}_{\text {inicial* }}^{*}$ & $\mathrm{pH}$ final $^{*}$ & $\mathrm{P}$ Value \\
\hline Grupo $A(N=9)$ & $7,03 \pm 0,71$ & $7,26 \pm 0,51$ & $0,2813 \mathrm{~T}$ \\
\hline Grupo $B(N=10)$ & $6,98 \pm 0,60$ & $6,97 \pm 0,29$ & $0,9575 \oplus$ \\
\hline
\end{tabular}

*Media \pm SD según número de participantes.T t-pareada, $\oplus$ Wilcoxon.

Tabla VI. Comparación del Flujo salival intergrupo al inicio y final del tratamiento.

\begin{tabular}{lccc}
\hline & Grupo $A(N=9)$ & $\begin{array}{c}\text { Grupo B } \\
(\mathrm{N}=10)\end{array}$ & P Value \\
\hline Flujo inicial ${ }^{*} \ddagger$ & $0,5 \pm 0,14$ & $0,86 \pm 0,29$ & $0,0065 \oplus$ \\
Flujo final ${ }^{*} \ddagger$ & $0,6 \pm 0,24$ & $0,97 \pm 0,26$ & $0,0092 \mathrm{~T}$
\end{tabular}

${ }^{*}$ Media \pm SD según numero de participantes,$\ddagger \mathrm{ml} / \mathrm{min}$. $\oplus$ MannWhitney test, $\mathrm{T}$ t-student

Tabla VII. Comparación del Flujo salival intragrupo al inicio y final del tratamiento.

\begin{tabular}{lccc}
\hline & Flujo inicial ${ }^{*} \ddagger$ & Flujo final ${ }^{*} \ddagger$ & $P$ Value \\
\hline Grupo $A(N=9)$ & $0,5 \pm 0,14$ & $0,6 \pm 0,24$ & $0,2622 \mathrm{~T}$ \\
Grupo B $(\mathrm{N}=10)$ & $0,86 \pm 0,29$ & $0,97 \pm 0,26$ & $0,2141 \oplus$
\end{tabular}

${ }^{*}$ Media \pm SD según número de participantes. $\ddagger \mathrm{ml} / \mathrm{min}$. T t-pareada, $\oplus$ Wilcoxon
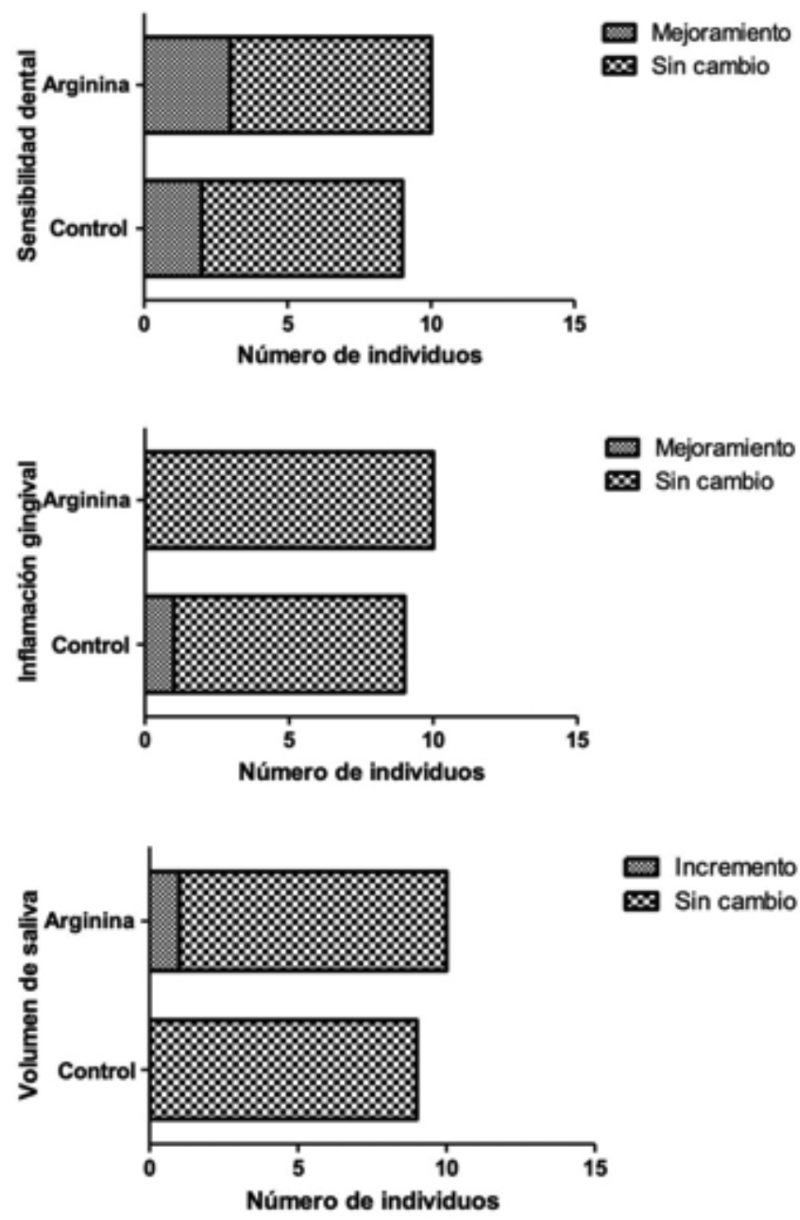

Incremento

$\infty$ Sin cambio
Con respecto al flujo salival la medición se muestra en la Tabla VI. Se identificó que había diferencias entre los grupos en la medición inicial y esta diferencia se mantuvo al finalizar el estudio. Por otro lado, al hacer el análisis intragrupo (Tabla VII) no se identificó un incremento significativo en el flujo salival.

Percepción de signos y síntomas orales antes y posterior a la utilización del gel: Se evaluaron signos y síntomas orales relacionados a sensibilidad dental, dolor, inflamación, sequedad y volumen de saliva previo y posterior a la utilización del gel. Se llevó a cabo un formulario en Google Forms y fue enviado a los participantes. En la Figura 2 se muestran los resultados de estas evaluaciones cualitativas. Ninguno de los parámetros cualitativos presentó una mejoría o cambio significativo en el grupo con gel con arginina al compararse con el grupo control el análisis estadístico se realizo con la prueba exacta de Fisher (valores p de 0,1409 a 1,0000).
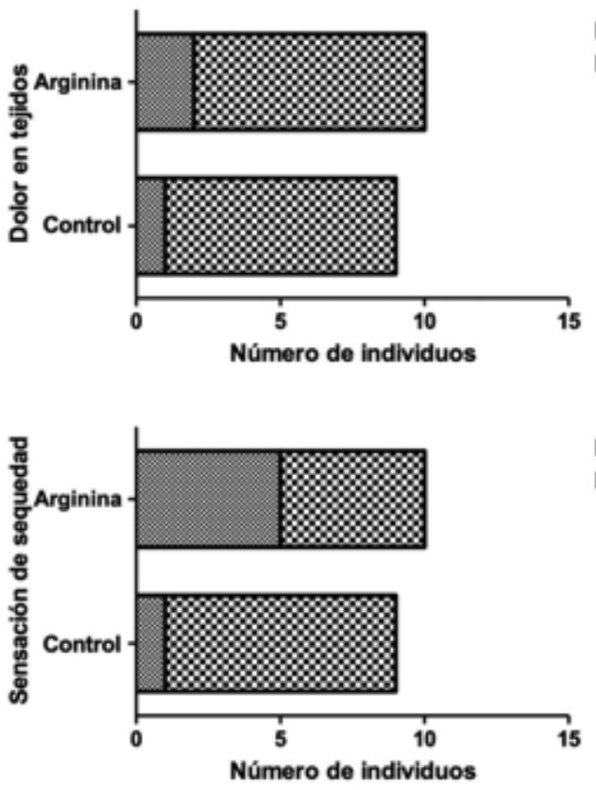

Mejoramiento $\infty$ Sin cambio

Fig. 2. Gráficos de Encuesta de Sintomatología Oral inicial y final. 


\section{DISCUSIÓN}

Este es el primer estudio realizado en México que evalúa la eficacia de un gel de arginina al $8 \%$ en el mantenimiento del $\mathrm{pH}$ y flujo salival en mujeres con desmineralización inicial tras su utilización durante dos semanas. Se encontró que no hubo diferencias estadísticamente significativas entre los grupos en el período de tiempo evaluado. En ambos grupos, el pH se encontró en valores neutros tanto en la evaluación inicial como en la final. A pesar de que ambos grupos presentaban un $\mathrm{pH}$ cercano a la neutralidad se identificó que existía desmineralización dental ICDAS 3, esto se explica por fluctuaciones que pueden ocurrir en el $\mathrm{pH}$ debido a la dieta.

Se sabe que el $\mathrm{pH}$ salival se mantiene cerca de la neutralidad debido a la arginina, ya que microorganismos arginolíticos de la cavidad oral la metabolizan por la vía ADS produciendo $\mathrm{NH} 4+$ (Nascimento; Bijle et al.; Monteagudo-Mera et al.). Sin embargo si en la composición del microbioma oral no predominan las bacterias arginolíticas si no las acidúricas debido a la poca ingesta de alimentos ricos en arginina, la neutralización del pH se dificultará y se tendrán períodos largos en $\mathrm{pH}$ críticos $<5.5$ los cuales favorecerán la pérdida de los minerales en los tejidos dentales (Wijeyeweera \& Kleinberg, 1989; Van Wuyckhuyse et al., 1995). En este grupo de pacientes la utilización de arginina tendría un efecto de mantenimiento en neutralizar el $\mathrm{pH}$ cuando exista acidificación en el microambiente oral. Estos resultados difieren de los reportados en un estudio realizado por Vuletic et al. en el cual evaluaron $\mathrm{pH}$ salival y cambio del flujo salival, ellos encontraron diferencias estadísticamente significativas con la utilización de la arginina en suplemento alimenticio durante una semana, estas diferencias pueden deberse a la vía de administración, ya que la arginina administrada por vía oral es absorbida rápidamente a la circulación y reciclada a la cavidad oral en donde permanece por más tiempo en el microambiente oral, y la colocación tópica en un hidrogel no logra que una cantidad suficiente sea absorbida para ser reciclada y mantener los niveles por más tiempo en el nicho (Vuletic et al.).

Por otro lado, la adición de la arginina en el grupo experimental y su obtención a través de la dieta en el grupo control fue suficiente para mantener el $\mathrm{pH}$ a valores cercanos a la neutralidad. Es importante mencionar que en el grupo experimental a pesar de obtener la arginina del hidrogel y de la dieta no se detecta- ron $\mathrm{pH}$ superiores a 7.3 ya que esto favorecería un microambiente favorable para el desarrollo de bacterias proteolíticas y por ende el desarrollo de una disbiosis periodontal, esto se debe a que el sistema arginolítico siempre va a mantener la neutralidad del $\mathrm{pH}$ siempre que las condiciones del microambiente oral sean las favorables como en este grupo de pacientes, donde la higiene oral fue buena (Huang et al., 2015; Esteban-Fernández et al., 2019).

Con respecto al flujo salival éste se encontraba en valores normales y con la intervención se logró un ligero aumento, aunque este no fue estadísticamente significativo, sin embargo, las pacientes desde la medición inicial mostraron un flujo salival adecuado lo cual influye de manera positiva al reducir la susceptibilidad de las estructuras orales a la desmineralización (Humphrey et al., 2001). Sin embargo, este efecto no se logró obtener en las pacientes ya que clínicamente había desmineralización, por lo que el pH puede jugar un papel más importante en mantener la homeostasia del microambiente oral que el flujo salival. Este ligero aumento del flujo salival se puede justificar a la colocación de un agente extraño en la cavidad oral (acetato) y a la naturaleza de la goma guar por lo que la Arginina no estaría involucrada en este cambio (Humphrey et al.; Dodds et al., 2015).

Referente a la sintomatología y signos orales los sujetos que utilizaron el hidrogel de arginina mencionaron tener una mejoría en algunos de los criterios evaluados, la sensibilidad dental, la inflamación gingival, dolor en tejidos blandos, el aumento del flujo salival. Los análisis estadísticos no arrojaron diferencias significativas, Sin embargo, esto podría ser evaluado en futuros estudios con una $n$ más grande.

La adición de arginina en pacientes con una desmineralización ICDAS 3 (esmalte y dentina) puede tener un beneficio en la remineralización dental, esto de acuerdo con lo publicado por Wijeyeweera \& Kleinberg.

Una de las fortalezas de este estudio fue la aplicación de la arginina en hidrogel al $8 \%$, esta dosis se seleccionó de acuerdo con diferentes estudios realizados con pastas dentales las cuales contienen Arginina al $8 \%$, y se obtuvieron resultados favorables para la prevención de caries (Zaura \& Twetman). Se sabe que el uso de la goma guar que es generalmente reconocido como seguro no genera ninguna interacción con la Arginina sin embargo hay un estudio que demuestra la capacidad de la goma guar de proteger la hidroxiapatita contra la acidez in vitro. Lo 
que no se sabe es si tiene un efecto sobre el $\mathrm{pH}$ y flujo salival (VanderReijden et al., 1997). El gel se formuló para contener la menor cantidad de aditivos posibles y así evitar sesgos como los que se asocian a productos comerciales. Se mantuvo estable en refrigeración $\left(4^{\circ} \mathrm{C}\right)$ por cerca de un mes y medio, después de este tiempo comenzaba a perder la consistencia gelatinosa. Probablemente la vida útil del gel sea más larga si se adicionan conservadores (Mudgil et al., 2014; Thombare et al., 2016).

Otra fortaleza del estudio radica en el hecho de que los criterios de inclusión fueron controlados para el control de las variables confusoras que podían influir en modificar la variable de respuesta $(\mathrm{pH}$ y flujo salival), por tal motivo solo se incluyeron pacientes del sexo femenino ya que el microambiente oral muestra diferencias con respecto al género (Zaura et al.; Sharma et al., 2018). Por otro lado, solo fueron incluidos sujetos con al menos un órgano dental ICDAS 3 , y con la presencia de pocas restauraciones, además su dieta, higiene, y vía de nacimiento fueron similares y no hubo diferencias entre los grupos (Vuletic et al.).

Con respecto a las limitaciones, una de las más importantes fue el tamaño de la muestra, sin embargo, se incluirán en futuros estudios más pacientes para verificar la hipótesis.La perspectiva de este trabajo es realizar este proyecto con pacientes en disbiosis oral con un microambiente predominantemente ácido y con ICDAS superiores a 3 , en los cuales la utilización de la arginina pueda mostrar un beneficio mayor. Además de incrementar el tiempo de aplicación, así como las tomas de muestras.

SÁNCHEZ, M. J. G.; ARAGÓN, M. O. H.; CEPEDA, B. J. A. \& ARANDA, R. S. Evaluation of the effect of arginine gel on $\mathrm{pH}$ and salivary flow. Int. J. Odontostomat., 15(4):989996, 2021.

ABSTRACT: The effect of an arginine gel on $\mathrm{pH}$ and salivary flow was evaluated after fourteen days of use in women with mild demineralization. A controlled pilot clinical trial was established in which 20 women aged 18-23 years, systemically healthy and with at least one dental organ with demineralization ICDAS 3 were included, two groups: Group A / without arginine $(N=10)$ and Group $B$ / with arginine $(N=$ 10). A clinical evaluation was carried out, and a sample of unstimulated saliva was taken to determine the $\mathrm{pH}$ and the measurement of salivary flow at the beginning and 15 days after using the gel. Statistical analysis was performed with the GraphPad Prism version 8 program. A $p<0.05$ was considered statistically significant. In both groups, salivary $\mathrm{pH}$ was maintained close to neutrality without statistically significant differences, and salivary flow remained at normal values after using the hydrogel for 14 days, although statistically significant differences were observed in the intergroup comparison. Using a gel with arginine for 14 days kept the neutral $\mathrm{pH}$ and salivary flow at normal levels without statistically significant differences from the control group.

KEY WORDS: caries, arginine, prebiotics, saliva, hydrogen-Ion concentration, flow rate.

\section{REFERENCIAS BIBLIOGRÁFICAS}

Abe, K.; Takahashi, A.; Fujita, M.; Imaizumi, H.; Hayashi, M.; Okai, K. \& Ohira, H. Dysbiosis of oral microbiota and its association with salivary immunological biomarkers in autoimmune liver disease. PloS One, 13(7):e0198757, 2018.

Bijle, M. N. A.; Ekambaram, M.; Lo, E. C. M. \& Yiu, C. K. Y. The combined antimicrobial effect of arginine and fluoride toothpaste. Sci. Rep., 9:8405, 2019.

Dodds, M.; Roland, S.; Edgar, M. \& Thornhill, M. Saliva A review of its role in maintaining oral health and preventing dental disease. BDJ Team, 2:15123, 2015.

Esteban-Fernández, A.; Ferrer, M. D.; Zorraquín-Peña, I.; LópezLópez, A.; Moreno-Arribas, M.V. \& Mira, A. In vitro beneficial effects of Streptococcus dentisani as potential oral probiotic for periodontal diseases. J. Periodontol., 90(11):1346-55, 2019.

Ferrer, M. D., López-López, A.; Nicolescu, T.; Salavert, A.; Méndez, I.; Cuñé, J.; Llena, C. \& Mira, A. A pilot study to assess oral colonization and $\mathrm{pH}$ buffering by the probiotic Streptococcus dentisani under different dosing regimens. Odontology, 108(2):180-7, 2019.

Huang, X.; Schulte, R. M.; Burne, R. A. \& Nascimento, M. M. Characterization of the arginolytic microflora provides insights into $\mathrm{pH}$ homeostasis in human oral biofilms. Caries Res., 49(2):165-76, 2015.

Humphrey S.P. \& Williamson R.T. A review of saliva: Normal composition, flow, and function. J. Prosthet. Dent., 85:162-9, 2001.

International Agency for Research on Cancer. Common Minimum Technical Standards and Protocols for Biobanks Dedicated to Cancer Research. Ginebra, Organización Mundial de la Salud, 2017. Disponible en: https://publications.iarc.fr/Book-And-ReportSeries/larc-Technical-Publications/Common-Minimum-TechnicalStandards-And-Protocols-For-Biobanks-Dedicated-To-CancerResearch-2017

Krishnan, K.; Chen, T. \& Paster, B. J. A practical guide to the oral microbiome and its relation to health and disease. Oral Dis., 23(3):276-86, 2017.

Lamont, R. J.; Koo, H. \& Hajishengallis, G. The oral microbiota: dynamic communities and host interactions. Nat. Rev. Microbiol., 16(12):745-59, 2018.

Lin, T. H.; Lin, C. H. \& Pan, T. M. The implication of probiotics in the prevention of dental caries. Appl. Microbiol. Biotechnol., 102(2):577-86, 2018.

Monteagudo-Mera, A.; Rastall, R. A.; Gibson, G. R.; Charalampopoulos, D. \& Chatzifragkou, A. Adhesion mechanisms mediated by probiotics and prebiotics and their potential impact on human health. Appl. Microbiol. Biotechnol., 103(16):6463-72, 2019.

Mudgil, D.; Barak, S. \& Khatkar, B. S. Guar gum: processing, properties and food applications-A Review. J. Food Sci. Technol., 51(3):409-18, 2014. 
Nascimento, M. M. Potential uses of arginine in dentistry. Adv. Dent. Res., 29(1):98-103, 2018.

Proclinic. KaVoDIAGNOdent $\circledast$ pen 2190 Display 2191. Una detección de la caries segura y sencilla. 2018. Disponible en: https:// www.proclinic.es/tienda/media/fichas_tecnicas/ diagnodent_if_es.pdf

Sharma, N.; Bhatia, S.; Sodhi, A. S. \& Batra, N. Oral microbiome and health. AIMS Microbiol., 4(1):42-66, 2018.

Thombare, N.; Jha, U.; Mishra, S. \& Siddiqui, M. Z. Guar gum as a promising starting material for diverse applications: A review. Int. J. Biol. Macromol., 88:361-72, 2016.

Van Wuyckhuyse, B. C.; Perinpanayagam, H. E. R.; Bevacqua, D.; Raubertas, R. E.; Billings, R. J.; Bowen, W. H. \& Tabak, L. A. Association of free arginine and lysine concentrations in human parotid saliva with caries experience. J. Dent. Res., 74(2):68690, 1995.

VanderReijden, W. A.; Buijs, M. J.; Damen, J. J.; Veerman, E. C.; ten Cate, J. M. \& Nieuw Amerongen, A. V. Influence of polymers for use in saliva substitutes on de- and remineralization of enamel in vitro. Caries Res., 31(3):216-23, 1997.

Vuletic, L.; Spalj, S.; Rogic, D.; Ruzic, L. \& Alajbeg, I. Effect of Larginine dietary supplementation on salivary urea concentration and $\mathrm{pH}$ in physically active individuals. Aust. Dent. J., 58(4):4917, 2013.

Wang, X. L.; Cheng, C. Y.; Peng, D.; Wang, B. \& Gan, Y. H. Dental plaque $\mathrm{pH}$ recovery effect of arginine bicarbonate rinse in vivo. Chin. J. Dent. Res., 15(12):115-20, 2012.

Wijeyeweera, R. L. \& Kleinberg, I. Arginolytic and ureolytic activities of pure cultures of human oral bacteria and their effects on the $\mathrm{pH}$ response of salivary sediment and dental plaque in vitro. Arch. Oral Biol., 34(1):43-53, 1989.

Zaura, E. \& Twetman, S. Critical appraisal of oral pre- and probiotics for caries prevention and care. Caries Res., 53(5):514-26, 2019.

Zaura, E.; Nicu, E. A.; Krom, B. P. \& Keijser, B. J. F. Acquiring and maintaining a normal oral microbiome: current perspective. Front. Cell. Infect. Microbiol., 4:85, 2014.

Zhan, L. Rebalancing the caries microbiome dysbiosis: targeted treatment and sugar alcohols. Adv. Dent. Res., 29(1):110-6, 2018.
Corresponding author:

Saray Aranda Romo

Departamento de Microbiología y Patología Oral Facultad de Estomatología

Universidad Autónoma de San Luis Potosí, S.L.P MÉXICO

E-mail: aran_sa77@hotmail.com 